\title{
Fatores de Risco e Riscos Laborais associados à aplicação e manutenção de Unhas Artificiais
}

\section{LABOR RISKS AND RISK FACTORS ASSOCIATED WITH ARTIFICIAL NAILS APPLICATION AND MAINTENANCE}

Autores: Santos, M(1); Almeida, A(2); Lopes C(3), Oliveira T(4) .

Tipo de estudo: Revisão Bibliográfica Integrativa ou Scoping Review

\section{RESUMO}

\section{Introdução e objetivos}

O uso de unhas artificiais surgiu há alguns anos e, dada a facilidade de acesso e a aceitabilidade estética das mesmas, a sua utilização tem sido cada vez mais prevalente, pelos que os os problemas médicos inerentes ao uso e produção das mesmas deverão tornar-se cada vez mais frequentes.

\section{Metodologia}

Trata-se de uma Scoping Review, elaborada em março de 2019, utilizando os motores de busca Scopus; PubMed; Web of Science; Science Direct; Academic Search Complete; CINALH; MedLine; Database of Abstracts and Reviews; Central Register of Controlled Trials; Cochrane Database of Systematic Reviews; Nursing and Allied Health Collection; MedicLatina e RCAAP.

\section{Conteúdo/ Resultados}

Recentemente têm surgido muitos casos de dermatite de contato alérgica aos acrilatos entre técnicas de unhas; estes estão presentes nos vernizes tradicionais, produtos para unhas de gel endurecidas por radiação ultravioleta e unhas acrílicas.

A alergia pode ser diagnosticada/ comprovada através de testes cutâneos. Estima-se que a sua prevalência seja na ordem de $1 \%$. Os profissionais deste setor justificam já $80 \%$ dos testes cutâneos para avaliação da dermatite de contato alérgica em contexto laboral. A sensibilização pode ocorrer até por via inalatória. 


\section{Discussão}

Não foram encontradas referências aos fatores de risco ruido, vibrações, radiações ultravioletas (que possam atingir o profissional), postura sentada mantida, eventuais movimentos repetitivos, bem como turnos prolongados e com poucas ou nenhumas pausas. Por sua vez, a nível de medidas de proteção coletiva, não foram encontradas quaisquer indicações e os Equipamentos de Proteção Individual mencionados não são suficientes. Para a questão específica dos acrilatos, encontram-se vários artigos associados a outros contextos profissionais.

\section{Limitações}

Os autores desenvolveram esforços no sentido de tentar que a sua pesquisa fosse exaustiva mas, uma vez concluída, perceberam que não encontraram dados relevantes sobre: doseamento de agentes químicos relevantes; indicação de quais técnicas são possíveis utilizar e quais as preferenciais; doseamento biológico (quando aplicáveis) numa amostra geral de profissionais do setor; referências ao tipo de amostra mais adequado a cada situação ou avaliação do risco global associado.

\section{Conclusões}

Os técnicos de unhas artificiais estão sujeitos a vários fatores de risco e riscos relevantes, para os quais não estão, por vezes, acauteladas as medidas de proteção coletiva e individual adequadas.

Seria muito pertinente que surgissem equipas motivadas para estudar melhor este setor e colmatar parte das limitações encontradas, não desenvolvidas na literatura internacional.

PALAVRAS-CHAVE: unhas artificiais, unhas de gel, unhas acrílicas, acrilatos, saúde ocupacional, medicina do trabalho.

\section{ABSTRACT}

\section{Introduction and objectives}

The use of artificial nails arose a few years ago and, given the ease access and the aesthetic acceptability, their use has been increasingly more prevalent, so the health problems inherent to the use and production of them should become more frequent also.

\section{Methodology}

This is a Scoping Review, written in March 2019, using the Scopus search engines; PubMed; Web of Science; Science Direct; Academic Search Complete; CINALH; MedLine; Database of Abstracts and Reviews; Central Register of Controlled Trials; Cochrane Database of Systematic Reviews; Nursing and Allied Health Collection; MedicLatina and RCAAP. 


\section{Content / Results}

Many cases of allergic contact dermatitis (ACD) to acrylates have recently appeared between nail technicians; these are present in traditional lacquers, ultraviolet-hardened gel nail products and acrylic nails.

This allergy can be diagnosed through skin tests. It is estimated that its prevalence is on the order of $1 \%$. The professionals of this sector already justify $80 \%$ of the skin tests for evaluation of the ACD in labor context. Sensitization may occur even by inhalation.

\section{Discussion}

No references were found to risk factors such as noise, vibration, ultraviolet radiation (for the professional), maintained seated posture, repetitive movements, as well as prolonged shifts and with few or no pauses. At the level of collective protection measures, no indications were found and the Individual Protection Equipment mentioned is not sufficient. For the specific question of acrylates, there are several articles associated with other professional contexts.

\section{Limitations}

The authors made efforts to try to make their research exhaustive but, once completed, they realized that they did not find relevant data on the determination of relevant chemical agents; indication of which techniques are possible and which are preferred to measure that in work environment or at a biological context (where applicable); references to the type of sample best suited to each situation or associated overall risk assessment.

\section{Conclusions}

Artificial nail technicians are subject to a number of relevant risk factors and risks, for which the appropriate collective and individual protection measures sometimes are not taken into account.

It would be very pertinent to have motivated teams to study this sector better and to fill some of the limitations found, not developed in the international literature.

KEYWORDS: artificial nails, gel nails, acrylic nails, acrylates, occupational health, occupational medicine.

\section{INTRODUÇÃO}

O uso de unhas artificiais surgiu há alguns anos e os materiais disponibilizados para esse efeito conseguiram seduzir a população feminina, que assim passou a conseguir ter unhas fortes, simétricas, longas e, no global, esteticamente mais agradáveis, a um preço acessível à generalidade da população. Dada a facilidade de acesso, a sua utilização tem vindo a evoluir exponencialmente e não é previsível um recuo neste consumo, pelos que todos os problemas médicos inerentes ao uso e produção das mesmas deverão tornar- 
se cada vez mais prevalentes; ainda que o foco deste artigo seja o contexto ocupacional, a análise pontual do que acontece aos consumidores do serviço, em alguns artigos, também poderá ser relevante.

\section{OBJETIVO}

Os autores tiveram como objetivo recolher e resumir toda a informação que encontraram sobre o tema, sob o formato de uma Scoping Review, como ponto de partida para outros projetos que se afirmem como pertinentes, no contexto da saúde ocupacional destes profissionais.

\section{METODOLOGIA}

Realizaram-se pesquisas informais prévias sobre o tema e percebeu-se que a literatura sobre fatores de risco/ riscos laborais é muito escassa para este setor profissional; por isso, os autores optaram por não fazer restrições significativas associadas a ano de publicação, tipo de estudo, robustez metodológica/ tipo de estudo, idioma ou acesso imediato a texto completo.

Como critérios de inclusão consideraram-se:

- publicação entre 1980 e 2019

- idade igual ou superior a 18 anos

- trabalho com vínculo formal ou informal ao setor.

Como critérios de exclusão foram assumidos:

estudos não pertinentes para o objetivo da revisão, ou seja, que não respondam à questão de investigação.

Foram considerados os seguintes motores de busca/ bases de dados: Scopus; PubMed; Web of Science; Science Direct; Academic Search Complete; CINALH; MedLine; Database of Abstracts and Reviews; Central Register of Controlled Trials; Cochrane Database of Systematic Reviews; Nursing and Allied Health Collection; MedicLatina e RCAAP.

Após análise da bibliografia dos documentos selecionados, houve a possibilidade de considerar os artigos aí mencionados, caso respondessem à pergunta de investigação. De igual forma, também se procuraram documentos publicados posteriormente, que tenham citado os inicialmente selecionados, de forma a avaliar se estes também poderiam dar algum contributo para elucidar os objetivos considerados.

A pergunta de investigação considerada foi: $\mathbf{O}$ que está descrito na literatura relativamente aos riscos ocupacionais dos Técnicos que elaboram unhas artificiais?

Nos dois quadros inseridos neste artigo, os autores sintetizaram as estratégias utilizadas para encontrar artigos pertinentes, nas diversas bases de dados/ motores de busca. 


\section{CONTEÚDO OU RESULTADOS}

\section{Tipos de unhas artificiais e evolução deste setor profissional}

As unhas de gel são obtidas através do uso de um verniz que, com a exposição a radiação ultravioleta adquire uma consistência endurecida (1) (2) (3) (4) (após polimerização), nomeadamente apenas com cerca de cinco minutos ou menos em cada mão ${ }^{(2)}$ e cujo efeito dura cerca de duas ${ }^{(1)}$ a três ${ }^{(2)}$ semanas. As peças em material acrílico polimerizam espontaneamente (3). As unhas artificiais têm sido cada vez mais usadas, devido à sua resistência e brilho ${ }^{(2)}$.

Alguns procedimentos na construção da unha implicam a aplicação de várias camadas e algum efeito decorativo superficial (5).

As empresas prestadoras destes serviços ungueais estão em crescimento exponencial em vários países (6) (7). Por exemplo, nos EUA, mais de 400.000 profissionais estão licenciados para trabalhar no setor. Logo, teremos cada vez mais indivíduos expostos a estes produtos ${ }^{(6)}$.

Não foram encontradas normas reguladoras para este setor e parte dos profissionais é inexperiente com as técnicas de trabalho em si e por vezes nem se apercebe dos riscos envolvidos (tal como grande parte dos consumidores) ${ }^{(1)}$ - seria pertinente que tal se alterasse $^{(5)}$.

\section{Riscos químicos para o profissional que elabora as unhas}

A dermatite de contato alérgica (DCA) secundária aos acrilatos e metacrilatos está bem documentada (3) (4) (8). O primeiro caso associado a unhas artificiais foi descrito em 1956, por Canizares (3) (4).

Mais recentemente, também se encontrou um caso clínico onde se considerou que estas substâncias conseguiram agravar uma urticária prévia, ainda que tal seja um facto inédito, em função dos conhecimentos prévios de quem escreveu esse artigo. A prevalência de DCA no global é de cerca de 20\%; se se restringir à região anatómica da mão, esse valor passa para 2\%; por sua vez, a urticária crónica terá a prevalência de cerca de $1,4 \%{ }^{(8)}$.

Recentemente têm surgido muitos casos de dermatite de contato alérgica aos acrilatos entre técnicas de unhas; estas substâncias estão presentes nos vernizes tradicionais (6), bem como nos produtos para unhas de gel endurecidas por radiação ultravioleta e unhas acrílicas ${ }^{(6)}$. Estes vernizes têm sido cada vez mais vendidos nos últimos anos, devido ao facto de serem inodoros e mais fáceis de manusear ${ }^{(8)}$.

A alergia pode ser diagnosticada/ comprovada através de testes dermatológicos cutâneos $(8)^{3.1}$. Estima-se que a sua prevalência seja na ordem de $1 \%{ }^{(1)}$. Os profissionais deste setor justificam já $80 \%$ dos testes cutâneos para avaliação da DCA em contexto 
laboral (6). A sensibilização pode ocorrer até por via inalatória (1). Contudo, uma vez polimerizados, estes compostos ficam razoavelmente inertes (1) (3). Ainda assim as reações cruzadas são razoavelmente frequentes ${ }^{(1)}(6)$.

Geralmente também está associado o formaldeído a estes compostos mas, nos testes cutâneos dermatológicos, ele não costuma ser relevante ${ }^{(6)}$.

Estes profissionais geralmente exercem em turnos prolongados e têm contato com vários agentes químicos que podem ser irritantes ou sensibilizadores, nomeadamente a nível respiratório. Além disso, estes locais de trabalho, por vezes, não têm ventilação adequada (ou esta simplesmente não existe) e, por isso, a qualidade do ar ambiente é inferior à desejável (9).

\section{Riscos considerados para as consumidoras destes serviços}

A alergia aos (met)acrilatos também tem vindo a aumentar na população consumidora destes serviços (1) (5), até porque cada vez mais se usam kits domiciliários para fazer unhas de gel (1) (3), colar unhas e colocar extensões de pestanas. Para além disso, alguns destes dispositivos têm também radiação ultravioleta (1). Está recomendado que se aplique um creme de proteção solar (para a cliente), mas raramente isso ocorre na realidade, porque essa recomendação quase nunca é dada (2). Alguns investigadores colocam a hipótese de a exposição a radiações UVA nestes procedimentos dê um risco acrescido de carcinoma escamoso cutâneo, contudo, a relevância parece ser superior para a cliente versus profissional. Ainda assim, mesmo que possível, considera-se que esse risco é reduzido, até para a cliente ${ }^{(7)}$.

As reações alérgicas neste contexto associam-se geralmente aos acrilatos, como já se mencionou. Contudo, um caso clínico, associado a kit usado em domicílio, encontrou também cobalto, que originou uma alergia (não ficando claro se este também poderá existir em contexto profissional), até porque parte dos produtos não têm a composição totalmente discriminada. Estes produtos são cada vez mais frequentes, dado o interesse crescente da população e a facilidade de encontrar vídeos na internet que explicam como fazer em casa. Neste caso específico, a semiologia caraterizou-se sobretudo por eczema periungueal e palmar pruriginoso, com início dois meses depois de ter feito o procedimento pela primeira vez. O afastamento do produto fez reverter totalmente a semiologia em quatro semanas ${ }^{(5)}$.

\section{Semiologia mais relevante}

A semiologia mais frequente caracteriza-se por (ainda que os sintomas possam surgir anos após o contato inicial): 
- alterações/ distrofia ungueais(3) (4) (8)

- espessamento ungueal (hiperqueratose) $)^{(6)(8)}$

- alteração da $\operatorname{cor}^{(8)}$

- perda ungueal(1)

- descolamento/ divisão da unha (onicólise)

- infeção cutânea periungueal (paroníquia)(1) (3) (6)

- eritema periungueal e cuticular(1)(3)(8)

- edema periungueal e cuticular(1)(8)

- alterações do crescimento(1) $^{(1)}$

- eritema digital(1)(6)

- eritema das mãos(3) (4) (6)

- eritema dos antebraços ${ }^{(6)}$

- fissuras dolorosas (pulpite) (1)(3) (6)

- dermografismo(8)

- dermatite facial(1) (6)

- eritema retroauricular e malar

- eritema cervical(6)

- eritema do dorso dos pés(6)

- sintomas respiratórios(1) (4)

- sibilos

- pieira

- maior suscetibilidade a infeções ${ }^{(9)}$

- erupção periorbitária(6)

- vesículas(3)

- $\operatorname{parestesias}^{(4)}$

- irritação ocular(9)

Contudo, após algumas semanas de afastamento, a semiologia pode regredir totalmente ${ }^{(4)}(8)$.

\section{EPI (equipamentos de proteção individual)}

Estes compostos penetram com alguma facilidade nas luvas de latex. O profissional poderá optar por usar dois pares ou então luvas de nitrilo, que conseguem dar proteção por cerca de uma hora (1) (ainda que alguns investigadores recomendem o uso de dois pares em simultâneo, mesmo neste material) ${ }^{(6)}$. As luvas mais seguras são construídas por trilaminado de polietileno (1) (6) (e funcionarão por cerca de quatro horas (1) (4)) mas, dado serem não elásticas e aderirem mal à mão, não são adequadas a estas tarefas laborais (1).

A nível de equipamentos de proteção está também recomendada a máscara (4).

\section{DISCUSSÃO}


Finalizada a análise dos artigos selecionados percebeu-se que o único fator de risco mencionado para esta classe profissional foi o químico e com grande destaque para os (met)acrilatos, ou seja, quase sem mencionar outros agentes presentes (as exceções foram o formaldeído e o cobalto).

Por isso, na literatura consultada não foram encontradas referências ao ruido eventualmente existente no posto de trabalho (sobretudo se o gabinete de trabalho estiver em open space no interior de um cabeleireiro), das vibrações de algumas das máquinas utilizadas, das radiações ultravioletas que possam atingir o profissional, da postura sentada mantida, eventuais movimentos repetitivos, bem como turnos prolongados e com poucas ou nenhumas pausas.

Mais recentemente surgiram instrumentos de trabalho que trocaram a luz ultravioleta por luzes led, cujas eventuais consequências médicas laborais não foram estudadas, ainda que tal não tenha sido mencionado na bibliografia selecionada.

Por sua vez, a nível de medidas de proteção coletiva, não foram encontradas quaisquer indicações; poder-se-á supor que neste contexto seria relevante:

- sugerir a estipulação de normas perante o tempo máximo recomendado para trabalhar sem pausas

- incentivar a rotatividade de tarefas entre diferentes profissionais (se em contexto de cabeleireiro e se os indivíduos assumirem cargos diferentes) ou pelo menos rotatividade a nível de técnicas/ materiais de construção da unha, para minimizar alguns fatores de risco laborais)

- divulgar e promover técnicas de Ginástica Laboral orientadas por algum funcionário ou pelo próprio

- estruturar regras de uso de EPIs (a nível de modelos, materiais e tempos de uso)

- fomentar condições para uma adequada ventilação do local de trabalho, definindo parâmetros mínimos a serem cumpridos neste contexto

- organizar um programa adequado de sessões formativas de temas relevantes a nível de saúde ocupacional pertinentes para este setor

- promover o acesso aos exames de Saúde Ocupacional com o Médico do Trabalho

- contratar serviços do Técnico de Segurança no Trabalho para avaliar as condições e o patamar de risco, incluindo a realização dos doseamentos que forem pertinentes.

Por sua vez, a nível de EPIs, não foram encontradas referências ao uso de farda/ bata/ avental, gorro ou equivalente e manguitos.

Os (met)acrilatos estão também presentes em tintas, próteses dentárias (1) (3) (4) (6) (8)e em produtos usados em procedimentos ortopédicos cirúrgicos (1) (3) (4) (8), bem como na produção de plásticos ${ }^{(3)}$ resistentes/ endurecidos ${ }^{(6)}$, indústria gráfica, tintas, adesivos e têxteis (3) e fibra de vidro ${ }^{(8)}$. Contudo, devido ao uso cada vez mais frequente das extensões ungueais, esta situação passou a ser mais frequente (1) (3) globalmente e mais frequente neste setor profissional, em contexto ocupacional (1). Ainda assim, o setor da medicina dentária corresponde a $45,2 \%$ dos casos de alergias aos acrilatos, a nível 
ocupacional (3). Paralelamente, os procedimentos médicos dentários e ortopédicos cirúrgicos serão cada vez mais frequentes no contexto de uma população mais envelhecida (1).

Quanto ao que está descrito a nível laboral, para outras classes profissionais, em relação aos acrilatos, encontram-se artigos que realçam os efeitos nocivos que estes podem ter a nível do setor da produção de tintas/ pinturas (10) (11) (12) (13), produção de colas (14), produção de plásticos ${ }^{(15)}$, dentária (12) (13) (14) (15) (16) (17) (18), ortopedia ${ }^{(14)(19)(20)}$ e produção de móveis ${ }^{(21)}$.

Para a generalidade dos restantes fatores de risco ocupacionais presentes neste setor, é muito abundante a literatura genérica para outras classes profissionais, pelo que não fará sentido destacar exemplos concretos a mesma.

\section{LIMITAÇÕES}

Os autores desenvolveram esforços no sentido de tentar que a sua pesquisa fosse exaustiva mas, uma vez concluída, perceberam que não encontraram dados relevantes sobre:

- doseamento de agentes químicos relevantes nestes ambientes de trabalho; nem indicação de quais técnicas são possíveis utilizar e quais as preferenciais

- doseamento biológico (quando aplicáveis) numa amostra geral de profissionais do setor, expostos a este agente; nem referências ao tipo de amostra mais adequado a cada situação

- avaliação do risco global associado, em função dos doseamentos obtidos e restante análise ao posto de trabalho

- descrição de medidas de proteção coletiva.

\section{CONCLUSÕES}

Os técnicos de unhas artificiais estão sujeitos a vários fatores de risco e riscos relevantes, para os quais não estão, por vezes, acauteladas as medidas de proteção coletiva e individual adequadas.

Seria muito pertinente que surgissem equipas motivadas para estudar melhor este setor e colmatar parte das limitações encontradas, não desenvolvidas na literatura internacional.

\section{AGRADECIMENTOS}

Não se aplicam.

\section{CONFLITO DE INTERESSES}

Não se aplicam. 
Não se aplica.

\section{BIBLIOGRAFIA}

1. Muttardi K, White I, Banarjee P. The burden of allergic contact dermatitis caused by acrylates. Contact Dermatitis. 2016, 75, 180-194. DOI:10.1111/cod.12578

2. Bollard S, Beecher S, Moriaty N, Kelly J, Reagan P, Potter S. Skin Cancer Risk and the use of UV nail lamps. The Australian College of Dermatologists. 2018, 348-349.

3. Lin Y, Tsai S, Yang C, Tseng Y, Chu C. Allergic contact dermatitis caused by acrylates in nail cosmetic products: case reports and review of the literatures. Dermatologica Sinica. 2018, 36, 218-221. DOI: 10.1016-7j.dsi.2018.05.001

4. Vasquez-Osorio I, Espasandín-Arias M, García-Gavin J, Fernández-Redondo V. Allergic contact dermatitis due to acrylates in acrylic gel nails: a report of 3 cases. Actas Dermosifiliograficas. 2014, 105, 430-432.

5. Guarneri F, Guarneri C, Cannavó S. Nail-art and cobalt allergy. Contact Dermatitis. $2010,62,320-321$.

6. DeKoven S, DeKoven J, Holness D. (Meth)Acrylate Occupational Contact Dermatitis in Nail Salon Workers: a case series. Journal of Cutaneous Medicine and Surgery. 2017, 21(4), 340-344. DOI: 10.1177/1203475417701420

7. Diffey $B$, The risk of squamous cell carcinoma in women from exposure to UVA lamps used in cosmetic nail treatment. British Journal of Dermatology. 2012, 167, 1175-1178. DOI: 10.1111/j1365-2138.201211107.x

8. Dickison P, Smith S. Itching for nail fashion: chronic urticaria and chronic hand dermatitis secondary to acrylate and methacrylate allergy in gel nail varnish. Clinical and Experimental Dermatology. 2018, 43, 50-53. DOI:10.1111/ced.13252

9. Reutman S, Rohs A, Clark J, Jonhson B, Sammons D, Toennis C et al. A Pilot Respiratory Health Assessment of Nail Techicians: Symptoms, Lung Function and Airway Inflamation. American Journal of Industrial Medicine. 2009, 52, 868-875. DOI: 10.1002/ajim20751

10. Nakamura M, Arima Y, Yoneda K, Nobuhara S, Miyachi Y. Occupational contact dermatitis from acrylic monomer in paint. Contact Dermatitis. 1999, 40(4), 228-229.

11. Gruvberger B, Bruze M, Almgren G. Occupational dermatoses in a plant producing binders for paints and glues. Contact Dermatitis. 1998, 38(2), 71-77.

12. Sasseville D. Acrylates in contact dermatitis. Dermatitis. 2012, 23(1), 6-16. DOI: 10.1097/Der.Ob013e31823d1b81

13. Arceo J, Barata A, Salazar L. Dermatitis de contacto alérgica a los acrilatos. Dermatologia Revista Mexicana. 2013, 57, 389-393.

14. Zaremba J, Kiec-Swierczysnka M, Krecisz B, Swierczynska-Machura D. Acrylates as a significant source of occupational and non-occupational contact allergy. Medycyna Pracy. 2004, 55(4), 357-361.

15. Walters G, Robertson A, Moore V, Burge P. Occupation asthma caused by acrilic compounds from shield surveillance (1989-2014). Occupational Medicine. 2017, 67(4), 282-289. DOI: 10.1093/occmed/kqx036 
16. Heratizaded A, Werfel T, Schubert S, Geier J. Contact sensitization in dental technicians with occupational contact dermatitis- Data of the Information Network of Departments of Dermatology (IUDK) 2001- 2015. Contact Dermatitis. 2018, 78(4), 266-273.

17. Minamoto $K$, Watanabe $T$, Diepgen T. Self-reported hand eczema among dental workers in Japan- a cross-sectional study. Contact Dermatitis. 2016, 75(4), 230-239. DOI: $10.1111 /$ cod. 12656

18. Kocak O, Gul U. Patch test results of the dental personel with contact dermatitis. Cutaneous and Ocular Toxicology. 2014, 33(4), 299-302. DOI: 10.3109/15569527.2013.866132

19. Thomas $P$, Schuh $A$, ring J, Thomsen M. Orthopedic Surgical implants and allergies. Hautarzt. 2008, 59(3), 220-229. DOI: 10.1007/s00105-007-1453-3

20. Fregert S. Occupational hazards of acrylate bone cement in orthopaedic surgery. Acta Orthopaedica Scandinavica. 1983, 54(6), 787-789.

21. Surakka J, Lindh T, Rosen G, Fischer T. Workers dermal exposure to UV curable acrylates in the furniture and Parquet industry. The Annals of Occupational Hygiene. 2000, 44(8), 635-644.

Quadro 1: Resumo dos dados mais relevantes associados à pesquisa nos motores de busca assinalados

\begin{tabular}{|c|c|c|c|c|c|}
\hline Motor de busca & $\begin{array}{l}\text { Password } \\
1-2 \text { e } \\
\text { seguintes, } \\
\text { caso } \\
\text { existam }\end{array}$ & Critérios & $\begin{array}{l}\mathrm{N}^{\circ} \text { de } \\
\text { documentos } \\
\text { obtidos }\end{array}$ & $\begin{array}{l}\mathbf{N}^{\circ} \text { da } \\
\text { pesquisa }\end{array}$ & $\begin{array}{l}\text { Pesquisa } \\
\text { efetuada } \\
\text { ou não }\end{array}$ \\
\hline EBSCO (CINALH, & Gel nails & \multirow[t]{13}{*}{ humano } & 12 & 1 & $\operatorname{sim}$ \\
\hline $\begin{array}{l}\text { Medline, } \\
\text { Database of }\end{array}$ & Nail & & 0 & 2 & não \\
\hline Abstracts and & extensions & & & & \\
\hline Reviews, Central & & & & & \\
\hline Register of & & & & & \\
\hline Controlled Trials, & & & & & \\
\hline Cochrane & & & & & \\
\hline Database of & & & & & \\
\hline Systematic & & & & & \\
\hline Reviews, Nursing & & & & & \\
\hline \& Allied Health & & & & & \\
\hline Collection e & & & & & \\
\hline MedicLatina) & & & & & \\
\hline
\end{tabular}




\begin{tabular}{|c|c|c|c|c|c|}
\hline \multirow[t]{2}{*}{ RECAAP } & Unhas de & \multirow{2}{*}{$\begin{array}{l}\text { pesquisa } \\
\text { avançada; } \\
\text { título }\end{array}$} & 0 & 3 & não \\
\hline & $\begin{array}{l}\text { Extensões } \\
\text { de unhas }\end{array}$ & & 0 & 4 & não \\
\hline \multirow[t]{4}{*}{ SCOPUS } & Gel nails & & 215 & 5 & não \\
\hline & \multicolumn{2}{|c|}{ And occupational } & 25 & 6 & $\operatorname{sim}$ \\
\hline & \multicolumn{2}{|c|}{ Extension nails } & 382 & 7 & não \\
\hline & \multicolumn{2}{|c|}{ And occupational } & 6 & 8 & $\operatorname{sim}$ \\
\hline \multirow{4}{*}{$\begin{array}{l}\text { Academic Search } \\
\text { Ultimate }\end{array}$} & Nails & \multirow[t]{2}{*}{ humano } & 11.020 & 9 & não \\
\hline & \multirow{2}{*}{\multicolumn{2}{|c|}{ Gel nails }} & 142 & 10 & não \\
\hline & & & 60 & 11 & $\operatorname{sim}$ \\
\hline & \multicolumn{2}{|l|}{$\begin{array}{l}\text { Extension } \\
\text { nails }\end{array}$} & 10 & 12 & $\operatorname{sim}$ \\
\hline \multirow[t]{6}{*}{ Science Direct } & \multicolumn{2}{|l|}{ Gel nails } & 5209 & 13 & não \\
\hline & \multicolumn{2}{|c|}{ and extensions } & 1.189 & 14 & não \\
\hline & \multicolumn{2}{|c|}{ and occupational } & 174 & 15 & não \\
\hline & \multicolumn{2}{|l|}{ and risks } & 160 & 16 & não \\
\hline & \multicolumn{2}{|c|}{ and medicine } & 118 & 17 & não \\
\hline & \multicolumn{2}{|l|}{ and health } & 109 & 18 & $\operatorname{sim}$ \\
\hline \multirow[t]{5}{*}{ Web of Science } & \multicolumn{2}{|l|}{ Gel nails } & 162 & 19 & não \\
\hline & \multicolumn{2}{|c|}{ and occupational } & 6 & 20 & $\operatorname{sim}$ \\
\hline & \multicolumn{2}{|c|}{ Extension nails } & 231 & 21 & não \\
\hline & \multicolumn{2}{|c|}{ and occupational } & 0 & 22 & não \\
\hline & \multicolumn{2}{|c|}{$\begin{array}{l}\text { Extension nails and } \\
\text { risks }\end{array}$} & 29 & 23 & $\operatorname{sim}$ \\
\hline
\end{tabular}

Quadro 2: Artigos selecionados de cada pesquisa 


\begin{tabular}{|c|c|c|c|c|}
\hline $\begin{array}{l}N^{0} \text { das } \\
\text { pesquisas } \\
\text { efetivadas em } \\
\text { que se } \\
\text { selecionou pelo } \\
\text { menos um } \\
\text { artigo }\end{array}$ & $\begin{array}{l}N^{\circ} \text { de artigos } \\
\text { selecionados após } \\
\text { a leitura do título e } \\
n^{\circ} \text { na pesquisa } \\
\text { interna }\end{array}$ & $\begin{array}{l}\mathrm{N}^{\circ} \text { de artigos } \\
\text { selecionados } \\
\text { após a leitura } \\
\text { do resumo }\end{array}$ & $\begin{array}{l}\text { Justificação } \\
\text { de } \\
\text { exclusão }\end{array}$ & $\begin{array}{l}\text { Inclusão e } \\
\text { codificação }\end{array}$ \\
\hline 1 & $6(4,5,6,8,10,12)$ & 6 & & 1.1 a 1.6 \\
\hline 6 & $4(1,6,7,8)$ & 1 & $\begin{array}{l}6,7 \text { e } 8= \\
1.1,1.2 \text { e } \\
1.3\end{array}$ & 6.1 \\
\hline 11 & $\begin{array}{l}6(2,12,22,23,41 \mathrm{e} \\
46)\end{array}$ & 3 & $\begin{array}{l}2,12 \text { e } 22 \\
\text { são } 1.1 \text { a } \\
1.3\end{array}$ & 11.1 a 11.3 \\
\hline 20 & 1 & 0 & $=6.1$ & \\
\hline
\end{tabular}

\section{(1)Mónica Santos}

Licenciada em Medicina; Especialista em Medicina Geral e Familiar; Mestre em Ciências do Desporto; Especialista em Medicina do Trabalho e Doutoranda em Segurança e Saúde Ocupacionais, na Faculdade de Engenharia da Universidade do Porto. Presentemente a exercer nas empresas Medicisforma, Servinecra e Securilabor; Diretora Clínica das empresas Quercia e Gliese; Diretora da Revista Portuguesa de Saúde Ocupacional on line. Endereços para correspondência: Rua Agostinho Fernando Oliveira Guedes, 42, 4420-009 Gondomar. E-mail: s_monica_santos@hotmail.com.

\section{(2)Armando Almeida}

Doutorado em Enfermagem; Mestre em Enfermagem Avançada; Especialista em Enfermagem Comunitária; Pós-graduado em Supervisão Clínica e em Sistemas de Informação em Enfermagem; Docente na Escola de Enfermagem (Porto), Instituto da Ciências da Saúde da Universidade Católica Portuguesa; Diretor Adjunto da Revista Portuguesa de Saúde Ocupacional on line. 4420-009 Gondomar. E-mail: aalmeida@porto.ucp.pt. Orcid nº000-0002-5329-0625.

\section{(3)Catarina Lopes}

Licenciada em Enfermagem, desde 2010, pela Escola Superior de Saúde Vale do Ave. A exercer funções na área da Saúde Ocupacional desde 2011 como Enfermeira do trabalho autorizada pela Direção Geral de Saúde, tendo sido a responsável pela gestão do departamento de Saúde Ocupacional de uma empresa prestadora de serviços externos 
durante 7 anos. Atualmente acumula funções como Enfermeira de Saúde Ocupacional e exerce como Enfermeira Generalista na SNS24. Encontra-se a frequentar o curso Técnico Superior de Segurança do Trabalho. 4715-028. Braga. E-mail: catarinafflopes@gmail.com

\section{(4)Tiago Oliveira}

Licenciado em Enfermagem pela Universidade Católica Portuguesa. Frequenta o curso de Técnico Superior de Segurança no Trabalho. Atualmente exerce a tempo inteiro como Enfermeiro do Trabalho. No âmbito desportivo desenvolveu competências no exercício de funções de Coordenador Comercial na empresa Academia Fitness Center, assim como de Enfermeiro pelo clube de futebol União Desportiva Valonguense. 4435-718 Baguim do Monte. E-mail: tiago_sc16@hotmail.com. 